\title{
Study of clinical, haematological and cytogenetic profile of patients with acute erythroid leukaemia
}

\author{
Jacob Abraham Linu, MS Namratha Udupa, DS Madhumathi, KC Lakshmaiah, K Govind Babu, D Lokanatha, MC Suresh Babu, \\ KN Lokesh, LK Rajeev and AH Rudresha
}

Kidwai Memorial Institute of Oncology, Bengaluru 560029, India

Correspondence to: MS Namratha Udupa. E-mail: msn.udupa@gmail.com

\section{Abstract}

Background: Acute erythroid leukaemia (AEL) is a rare subtype of acute myeloid leukaemia (AML), constituting $<5 \%$ of all the cases of AML. The World Health Organization (WHO) in 2001 classified AEL into two types: (1) erythroid/myeloid leukaemia which required $\geq 50 \%$ erythroid precursors with $\geq 20 \%$ of the non-erythroid cells to be myeloid blasts and (2) pure erythroleukemia ( $p E L$ ) with $\geq 80 \%$ erythroblasts. The WHO 2008 classification kept these subcategories, but made erythroleukemia a diagnosis of exclusion. There are very few studies on the clinico haematological and cytogenetic profile of this disease, considering the rarity of its occurrence and poor prognosis.

Materials and methods: This study was done by retrospective analysis of data from 32 case files of patients diagnosed with AEL. Clinical details noted down were the demographic profile, peripheral blood smear details and bone marrow examination details: (1) blasts-erythroblasts and myeloblasts, (2) dysplasia in the cell lineages and (3) cytogenetic abnormalities.

Results: The most common presenting symptom was fever. Pancytopenia at presentation was seen in $81.25 \%$ of patients. Dysplasia was observed in bone marrow in $100 \%$ of erythroblasts and in $40 \%$ of myeloblasts in erythroid/myeloid subtype. In pure myeloid subtype, myeloid and megakaryocytic dysplasias were not obvious. Complex karyotype was noticed only in patients of $\mathrm{pEL}$.

Conclusion: $\mathrm{AEL}$ is a rare group of heterogeneous diseases with many neoplastic and non-neoplastic conditions mimicking the diagnosis. The clinical presentation and cytogenetics are also non-specific, presenting additional challenges to the diagnosis.

Keywords: clinicohematological profile, cytogenetics, acute erythroid leukemia

Published: $10 / 01 / 2017$

Received: $27 / 08 / 2016$

ecancer 2017, 11:712 DOI: 10.3332/ecancer.2017.712

Copyright: (c) the authors; licensee ecancermedicalscience. This is an Open Access article distributed under the terms of the Creative Commons Attribution License (http://creativecommons.org/licenses/by/3.0), which permits unrestricted use, distribution, and reproduction in any medium, provided the original work is properly cited. 


\section{Background}

Acute erythroid leukaemia (AEL) is a rare subtype of acute myeloid leukaemia (AML), constituting $<5 \%$ of all the cases of $A M L$ [1]. The definition of this disease has been revised multiple times. The probability of existence of this disease was first deduced by Coppelli in 1912. He documented the first case of AEL [2]. In 1928, Di Guglielmo described the first case of pure erythroid leukaemia and documented it as eritremia acuta, which was later designated as Di Guglielmo disease [3]. Probability of pathologic evolution of acute erythroid myeloid leukaemia was first hypothesised by Damshek as development from phase of non-malignant erythremic proliferation through phase of erythroleukemia to myeloblastic leukaemia, and he named it Di Guglielmo syndrome [4].

AEL was designated as AML-M6 by the FAB Cooperative Group in 1976 and diagnosis required presence of erythroid precursors $\geq 30 \%$ and dyserythropoiesis $\geq 10 \%$. Following the amendments brought to the FAB classification in 1985, AML-M6 was to be defined by the criteria: $\geq 50 \%$ erythroblast of all nucleated cells, prominent dyserythropoiesis and $\geq 30 \%$ of myeloblast of the rest of non-erythroid precursors. In this, paradoxically ignored was the rare subtype of pure erythroid leukaemia which was classified under refractory anaemia with excess blasts in transformation [5]. The World Health Organization (WHO) in 2001 classified AEL into two types: (1) erythroid/myeloid leukaemia which required $\geq 50 \%$ erythroid precursors with $\geq 20 \%$ of the non-erythroid cells to be myeloid blasts and (2) pure erythroleukemia ( $p E L$ ) with $\geq 80 \%$ erythroblasts. WHO 2008 classification kept these subcategories, but made erythroleukemia a diagnosis of exclusion [2]. Despite these amendments to the definition, there is considerable overlap with the diagnosis of AEL with various myelodysplastic syndromes (MDSs) [6]. The latest amendment to the WHO classification in the year 2016 was a big stroke to the all the confusions surrounding this entity. The entity of AEL is being proposed to be removed [7-9]. The myeloblasts need to be counted as a percentage of the total marrow cells. This will be mostly $<20 \%$ and will qualify to be called as MDS, mostly the subtype with excess blasts. This study was made keeping in line with the 2008 classification as the 2016 classification was too recent at the time of conducting this study.

There are very few studies on the clinico haematological and cytogenetic profile of this disease, considering the rarity of its occurrence and poor prognosis.

\section{Materials and methods}

This study was done by retrospective analysis of data from case files of patients diagnosed with AEL at the Kidwai Memorial Institute of Oncology over a period of 19 years from January 1997 to March 2016. A total of 1223 of AML were diagnosed in this period of which 32 patients had AEL (2.6\%).

Clinical details noted down were the demographic profile: (1) age, (2) gender, (3) presenting symptoms and signs with duration, (4) past history of MDS/MPN or exposure to chemotherapy/radiotherapy and (5) family history. Also noted down were the peripheral blood smear details and bone marrow examination details: (1) blasts-erythroblasts and myeloblasts, (2) dysplasia in the cell lineages and (3) cytogenetic abnormalities. Peripheral smear and bone marrow aspiration slides of all the patients were reviewed, and diagnosis was revisited in view of new amendments to WHO definition of the entity. Diagnosis of AEL was made according to the WHO 2008 definition. Cytogenetic analysis was performed by conventional karyotyping of at least 10 metaphases, and the nomenclature system adopted was the International System for Chromosome Nomenclature (1985 and 1995).

\section{Results}

\subsection{Clinical profile}

32 AEL patients were diagnosed in this period in 19 years of which 28 were of myeloid/erythroid subtype (erythroleukemia-M6a) and 4 were of pure erythroid subtype (pEL-M6b). Of these patients, 22(68.75\%) were males and 10(31.25\%) were females. In the group of patients with $\mathrm{pEL}$, three were males and only one was a female patient. Median age was 36.3 years (Table 1). 
Table 1. Clinical Profile, Peripheral Smear and Bone Marrow Morphology.

\begin{tabular}{|l|l|l|}
\hline \multicolumn{1}{|c|}{ Clinical Characteristics } & \multicolumn{1}{c|}{ AEL } & \multicolumn{1}{c|}{ pEL } \\
\hline Median age & 36.3 years (27-51) & 42 years (35-67) \\
\hline Gender distribution & 22 males: 10 females: (2.2:1-M:F) & 3 males: 1 female:: 3:1 \\
\hline History of previous chemo/radiotherapy & None & None \\
\hline Family history & None & None \\
\hline Median leucocyte count & $5100 / \mathrm{mm}^{3}(3300-19200)$ & $2900 / \mathrm{mm}^{3}(1800-12000)$ \\
\hline Median neutrophil count & $2200(2000-8000)$ & $1500(1100-5000)$ \\
\hline Median platelet count & $95000 / \mathrm{mm}^{3}(30000-1.8$ lacs) & $52000 / \mathrm{mm}^{3}(10000-70000)$ \\
\hline Median haemoglobin & $8.2 \mathrm{~g} / \mathrm{dl}(5.5-11.2)$ & $6.5 \mathrm{~g} / \mathrm{dl}(4-9.2)$ \\
\hline Median bone marrow erythroblast percentage & 65 & 88 \\
\hline $\begin{array}{l}\text { Median bone marrow myeloblast percentage } \\
\text { (from total nucleated cells) }\end{array}$ & 15 & 10 \\
\hline $\begin{array}{l}\text { Median bone marrow myeloblast percentage } \\
\text { (from non-erythroid cells) }\end{array}$ & 25 & 20 \\
\hline Percentage with erythroid dysplasia & 100 & 100 \\
\hline Percentage with granulocytic dysplasia & 40 & 10 \\
\hline Percentage with megakaryocytic dysplasia & 30 & 0 \\
\hline
\end{tabular}

Table 2. Symptoms and Physical Findings.

\begin{tabular}{|l|c|}
\hline \multicolumn{1}{|c|}{$\begin{array}{c}\text { Presenting Symptom/ } \\
\text { Signs }\end{array}$} & Prevalence (\%) \\
\hline Fatigue & 87.5 \\
\hline Pallor & 78.12 \\
\hline Fever & 68.75 \\
\hline Generalised myalgia & 40 \\
\hline Jaundice & 31.25 \\
\hline Mucocutaneous bleeding & 15.6 \\
\hline Hepatomegaly & 15.6 \\
\hline Lymphadenopathy & 12.5 \\
\hline Splenomegaly & 9.3 \\
\hline Hepatosplenomegaly & 6.25 \\
\hline
\end{tabular}

The most common presenting symptom was fever seen in $22(68.75 \%)$ patients, whereas the most common symptom present was easy fatiguability seen in $28(87.5 \%)$ patients. Other prominent presenting symptoms were mild mucocutaneous bleeding seen in $5(15.6 \%)$ patients and lymphadenopathy seen in $4(12.5 \%)$ patients. Signs on examination were pallor in $25(78.12 \%)$ patients, jaundice in $10(31.25 \%)$ patients and organomegaly in $10(31.25 \%)$ patients: hepatosplenomegaly in $2(6.25 \%)$, only hepatomegaly in $5(15.62 \%)$ and only splenomegaly in 3(9.3\%) patients. Most patients had an ECOG PS of 2. At presentation, most of them required blood/component transfusions. Mean total bilirubin was $2.7 \mathrm{~g} / \mathrm{dl}(0.8-4.7)$ (Table 2). 


\subsection{Haematological profile}

\subsubsection{Peripheral smear}

Median haemoglobin was $7.8 \mathrm{~g} / \mathrm{dl}(4-11.2)$, median total leucocyte count was 4400/mm 3 (1800-19200) and median platelet count was $92000 / \mathrm{mm}^{3}$ (10000-1.8 lacs). Pancytopenia at presentation was seen in $81.25 \%$ of patients.

\subsubsection{Bone marrow morphology}

Bone marrow aspirate morphological examination had a mean erythroblast count of $65 \%(55-95)$, prominent shift to left with various dysplastic features: megaloblastic forms (22 patients-68.75\%), nuclear budding (19 patients-59.37\%), bi and multinucleated forms (21 patients-65.6\%), cytoplasmic vacuolization (17 patients-53.1\%), nuclear bridging (12 patients-37.5\%) and karyorrhexis (7 patients-21.8) (Table 3). Erythroid precursor dysplasia was observed in $100 \%$ of cases, whereas granulocytic and megakaryocytic dysplasias were seen in $40 \%$ and $30 \%$ of patients, respectively, in erythroid/myeloid subtype. In pure erythroid subtype, granulocytic and megakaryocytic dysplasias were not obvious (Figures 1-4).

Table 3. Dysplasia in Bone Marrow Morphology.

\begin{tabular}{|l|c|}
\hline \multicolumn{1}{|c|}{ Dysplastic Features } & Prevalence (\%) \\
\hline Erythroid dysplasia-Megaloblastic forms & 68.75 \\
\hline Erythroid dysplasia-Bi and multinucleated forms & 65.6 \\
\hline Erythroid dysplasia-Nuclear budding & 59.37 \\
\hline Erythroid dysplasia-Cytoplasmic vacuolization & 53.1 \\
\hline Erythroid dysplasia-Nuclear bridging & 37.5 \\
\hline Erythroid dysplasia-Karyorrhexis & 21.8 \\
\hline $\begin{array}{l}\text { Granulocytic dysplasia-hypolobated, (this is not a dysplastic trait), } \\
\text { hypo and degranulated neutrophils }\end{array}$ & 40 \\
\hline Megakaryocytic dysplasia-small in size, hypo and monolobulated & 30 \\
\hline
\end{tabular}

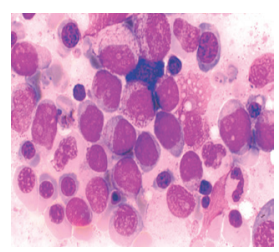

Figure 1. Hyperplastic marrow showing erythroblasts at all stages of maturation and are more than $50 \%$ of marrow cells. Myeloblasts are also present.

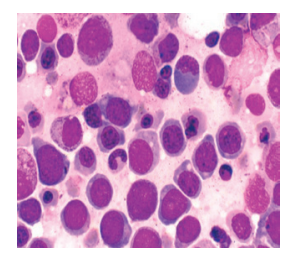

Figure 2. Hyperplastic marrow showing dysplasia of erythroblasts-macronormoblastic reaction, bi/multinucleated forms and karryorhexis. 


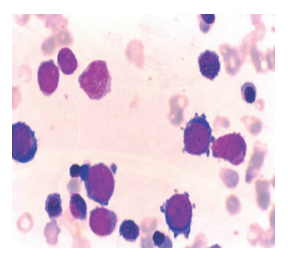

Figure 3. Erythroblasts constituting more than $\mathbf{8 0} \%$ of cells in the marrow-pure erythroid leukemia.

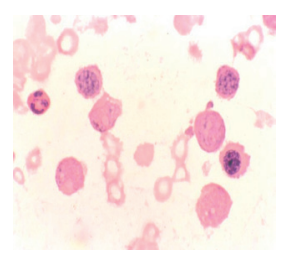

Figure 4. Erythroblasts demonstrating coarse granules on PAS staining.

Table 4. Cytogenetic Profile.

\begin{tabular}{|l|c|}
\hline \multicolumn{1}{|c|}{ Cytogenetics } & Prevalence \\
\hline Normal karyotype & $15(46.8)$ \\
10 patients: 46,XY & \\
5 patients: 46,XX & $3(9.3)$ \\
\hline 2 patients: 46,XY(-20) & \\
1 patient: 46,XX(-20) & $3(9.3)$ \\
\hline 3 patients: 46,XY/t $(3,5)$ & $1(3.12)$ \\
\hline 1 patient: $47, \mathrm{XX} /$ Trisomy 13 & $1(3.12)$ \\
\hline 1 patient: 47,XY/Trisomy 8 & $4(12.5)$ \\
\hline $\begin{array}{l}\text { Complex karyotype } \\
46, \mathrm{XY}(-3,-5,-11,13)\end{array}$ \\
$46, \mathrm{XY}(-3,+13,-20)$ & \\
$46, \mathrm{XY}(-9,-11,-20) / \mathrm{t}(3,5)$ & \\
$46, \mathrm{XX}(-15,-11,-20)$ & $5(15.4)$ \\
\hline No information available (Failed cytogenetics) & \\
\hline
\end{tabular}

\subsubsection{Cytogenetic profile}

Results of cytogenetics by conventional karyotyping is as detailed below. Complex karyotype was noticed only in patients of pEL (Table 4).

\section{Discussion}

AEL is a hematopoietic neoplasm affecting the erythroid and myeloid precursor cells of bone marrow. The definition of AEL has undergone metamorphoses in accordance with the expanding knowledge of various other disease entities which are pathological mimics of this disease and the biologic and clinical behaviour of the disease. The diagnosis of AEL is one of exclusion. The entities to be excluded are AML-myelodysplasia-related changes (AML-MRC), therapy-related AML (t-AML), AML with erythroblast proliferation and recurrent genetic abnormalities, erythroblast phase of myeloproliferative neoplasms (MPNs) and reactive erythroid hyperplasia after erythropoietin (EPO) treatment [2]. 
Diagnosis of AML-MRC requires the presence of at least $20 \%$ of blasts in the blood/marrow with one of the following: history of MDS/MPN, MDS-related cytogenetic abnormalities and absence of any of recurrent cytogenetic abnormalities, or presence of a minimum of $50 \%$ of dysplastic cells in at least two lineages with absence of history of exposure to cytotoxic agents: (1) alkylating agents and (2) topoisomerase II inhibitors [10]. t-AML has a history of exposure to alkylating agents/topoisomerase II inhibitors, specific cytogenetic abnormalities and a median time to development of 3-5 years [11]. Some MPNs are associated with erythroblastic phase characterised by marked erythroid hyperplasia of the bone marrow with nucleated RBCs in the peripheral blood [12]. There are other non-neoplastic differential diagnoses which need to be ruled in certain situations. These are EPO treatment, vitamin B12 or folate deficiency, exposure to toxins like benzene and parvovirus infection [2, 13]. EPO treatment can mimic pure erythroid leukaemia especially. One of the major differentiating features of the two, apart from history of treatment with EPO, would be that pure erythroid leukaemia presents with severe anaemia and circulating blasts whereas EPO-treated patients will have their anaemia corrected.

Incidence of AEL in our population of AML patients was $2.6 \%$. This is less than the incidence (4.3\%) quoted in Attili et al from the same institute [14]. In this, the share of pEL was $12.5 \%$. There was not even a single case of secondary AML. Various studies [15-18] have quoted similar incidence. Median age in our patients was 36.3 years, which is far lesser than age (56-66 years) in various other studies [15, 16]. Some studies have also quoted bimodal distribution of age $[6,19,20]$. The first small peak is found to be below the age of 20 years. We hypothesised this difference from other literature for two reasons. First, the median age incidence of all unclassified types of AML from an unpublished data from this institute is 37 years, which is quite an alarming development. There is also evidence for the earlier occurrence of other cancers like breast cancer in India [21]. Whether we can attribute this probable age shift to rapid urbanisation is an open-ended question which calls for further studies. Second, there was no case of secondary AEL which has an incidence of $20 \%-30 \%$ otherwise among secondary AMLs [14]. The disease has a male preponderance akin to the results from the other studies [15, 16, 19].

Most common presenting symptom was fever which was similar to that in other studies [18, 22]. AEL can present with non-specific clinical features [6, 14]. Symptoms could be due to anaemia and resultant asthenia [20]. Mean haemoglobin in our study was $7.8 \mathrm{~g} / \mathrm{dl}$ which was consistent with other studies $[6,18]$. Up to $30 \%$ of patients can have bleeding symptoms or organomegaly at presentation [6, 23]. However, some studies have incidence of organomegaly up to $70 \%$ [17]. In this series, organomegaly was seen in $15.6 \%$. Lymphadenopathy was seen in $12.5 \%$ of patients which is different from the absence of it in other studies [17-19]. One surprising feature was the occurrence of jaundice in about $31.25 \%$ of all AEL patients, but in all of the four patients of pEL. This is rarely though reported in other studies [24, 25]. Pancytopenia was seen in $81.25 \%$ of patients in this study which was higher than that in other studies. Up to $50 \%$ of patients have no blasts on peripheral smear [20]. Bleeding manifestations are reported in about one-third of patients [20, 26]. In this series, only minor mucocutaneous bleeding was noticed in $15 \%$ of patients.

Bone marrow was hypercellular with prominent dysplasia in erythroid lineage [20, 27]. In the presence of prominent dysplasia, erythroid prominent MDS (with $>50 \%$ erythroblasts) and AML-MRC are two closely related entities. Patients of MDS, in general, have anaemia and dysplasia which depicts a stress on the bone marrow. Diagnosis of MDS requires a blast percentage of $<20 \%$ of all nucleated cells and also $<20 \%$ of non-erythroid precursors [6]. The latter is to alleviate the risk of over-diagnosis of low blast count subset of AEL, which behaves more like MDS than AEL. This is a small subset of patients with high erythroid precursors $(70 \%-90 \%)$ which allows the myeloblasts to be $>20 \%$ of the non-erythroid precursors $[1,6]$. In addition, it has been shown that enumerating blasts as a percentage of non-erythroid cells are superior as compared with the percentage of all nucleated cells in risk stratification of the patients [28, 29]. At least one cell lineage dysplasia was seen in all the cases, most common being in erythroid lineage followed by megakaryocyte and myeloid lineages. Single lineage dysplasia was observed in $12(37.5 \%)$, double lineage in $15(46.8 \%)$ and triple lineage in $5(15.6 \%)$ of patients. In pure erythroid subtype, dysplasia in non-erythroid lineages was a minor finding. Only dysplasia in erythroid lineage was seen in eight patients (25\%), which is higher than that (10\%) observed in the study by Domingo-Claros et al [30]. Changes were non-specific, and megaloblastic forms were most commonly seen.

No cytogenetic abnormality is specific for AEL. Four patients with cytogenetic abnormalities involving deletions in chromosomes 5 and 7 , who were earlier classified as AEL from a study at the same institute [14], were reclassified as AML-MRC in the wake of new and more stringent definition of WHO 2008. However, all four patients of pure erythroid subtype harboured complex karyotype. This is similar to other articles on pure erythroid leukaemia [31, 32]. Complex karyotype was defined as having at least three cytogenetic abnormalities, but only in the absence of WHO termed recurrent inversions or translocations [33]. This could probably be explained by the younger patient population in this study, and it is known that the incidence of complex karyotype increases with age at diagnosis [34]. 
Of the 32 patients studied, $21(65 \%)$ received only the best supportive care; $4(12.5 \%)$ received low dose subcutaneous cytarabine; $3(9.3 \%)$ received decitabine; 4 received intensive $3+7$ induction chemotherapy of infusional daunorubicin and cytarabine. The overall survivals in the respective subgroups were 2, 4, 5 and 14 months. The median follow-up period was 7 months.

\section{Conclusion}

AEL is a rare group of heterogeneous diseases with many neoplastic and non-neoplastic conditions mimicking the diagnosis. The clinical presentation and cytogenetics are also non-specific offering additional challenges to the diagnosis. Though it has an inherent biological relationship with MDSs, it has a worse clinical behaviour. Amidst the thoughts of phasing out this diagnosis and merging it with MDS, one must appreciate its differences in clinical outcomes. A probable age shift with regard to a younger population calls for more studies in this regard.

\section{Conflict of interest}

None.

\section{References}

1. Hasserjian RP et al (2010) Acute erythroid leukemia: a reassessment using criteria refined in the 2008 WHO classification Blood 115(10) 1985-92 DOI: 10.1182/blood-2009-09-243964 PMCID: 2942006

2. Mihova D and Zhang L (2012) Acute erythroid leukemia: a review N Am J Med Sci 5(2) 110-8 DOI: 10.7156/v5i2p110

3. Bain BJ (2003) Di Guglielmo and his syndromes Br J Haematol 120(6) 939-43 DOI: 10.1046/j.1365-2141.2003.04181.x PMID: 12648062

4. Dameshek W (1958) Pernicious anemia, megaloblastosis and the di Guglielmo syndrome Blood 13(11) 1085-8 PMID: 13584478

5. Bennett JM et al (1985) Proposed revised criteria for the classification of acute myeloid leukemia. A report of the FrenchAmerican-British Cooperative Group Ann Intern Med 103(4) 620-5 DOI: 10.7326/0003-4819-103-4-620 PMID: $\underline{3862359}$

6. Zuo Z et al (2010) Acute erythroid leukemia Arch Pathol Lab Med 134(9) 1261-70 PMID: 20807044

7. Arber DA et al (2016) The $\mathbf{2 0 1 6}$ revision to the World Health Organization classification of myeloid neoplasms and acute leukemia Blood 127(20) 2391-405 DOI: 10.1182/blood-2016-03-643544 PMID: 27069254

8. Calvo X et al (2016) Erythroleukemia shares biological features and outcome with myelodysplastic syndromes with excess blasts: a rationale for its inclusion into future classifications of myelodysplastic syndromes Mod pathol: official journal of the United States and Canadian Academy of Pathology, Inc 29(12) 1541-51 DOI: 10.1038/modpathol.2016.146

9. Wang SA et al (2016) Acute erythroid leukemia with $<\mathbf{2 0} \%$ bone marrow blasts is clinically and biologically similar to myelodysplastic syndrome with excess blasts Mod Pathol: official journal of the United States and Canadian Academy of Pathology, Inc 29(10) 1221-31 DOI: 10.1038/modpathol.2016.118

10. Vardiman J and Reichard K (2015) Acute myeloid leukemia with myelodysplasia-related changes Am J Clin Pathol 144(1) 29-43 DOI: 10.1309/AJCP58RSMFRHLHHH PMID: $\underline{26071460}$

11. Bhatia S (2013) Therapy-related myelodysplasia and acute myeloid leukemia Semin Oncol 40(6) 666-75 DOI: 10.1053/j.seminoncol.2013.09.013 PMID: 24331189 PMCID: $\underline{3867743}$ 
12. Kojima $S$ et al (1987) Erythropoiesis during an erythroblastic phase of chronic myeloproliferative disorder associated with monosomy 7 Br J Haematol 65(4) 391-4 DOI: 10.1111/j.1365-2141.1987.tb04138.x PMID: 3580297

13. Moharram L et al (2014) Erythropoietin-induced acute erythroid leukemia-like picture: a potential pitfall Hematol Oncol Stem Cell Ther 7(1) 50-2 DOI: 10.1016/j.hemonc.2013.09.004

14. Attili SV et al (2006) A retrospective study of clinico-hematological and cytogenetic profile of erythroleukemia from South India Turk J Haematol: official journal of Turkish Society of Haematology 23(3) 158-63

15. Wells AW et al (2001) Erythroleukaemia in the north of England: a population based study J Clin Pathol 54(8) 608-12 DOI: 10.1136/jcp.54.8.608 PMID: $\underline{11477115}$ PMCID: $\underline{1731487}$

16. Hetzel P and Gee TS (1978) A new observation in the clinical spectrums of erythroleukemia. a report of 46 cases Am J Med. 64(5) 765-72 DOI: 10.1016/0002-9343(78)90515-6 PMID: $\underline{306198}$

17. Mazzella FM et al (1998) Acute erythroleukemia: evaluation of $\mathbf{4 8}$ cases with reference to classification, cell proliferation, cytogenetics, and prognosis Am J Clin Pathol 110(5) 590-8 DOI: 10.1093/ajcp/110.5.590 PMID: 9802343

18. Olopade $\mathrm{O}$ et al (1992) Clinical, morphologic, and cytogenetic characteristics of 26 patients with acute erythroblastic leukemia Blood 80(11) 2873-82 PMID: 1450412

19. Kowal-Vern A et al (2000) Diagnosis and characterization of acute erythroleukemia subsets by determining the percentages of myeloblasts and proerythroblasts in 69 cases Am J Hematol 65(1) 5-13 PMID: 10936857

20. Park S et al (2002) Erythroleukemia: a need for a new definition Leukemia 16(8) 1399-401 DOI: 10.1038/sj.leu.2402549 PMID: $\underline{12145675}$

21. Chopra B et al (2014) Age shift: Breast cancer is occurring in younger age groups - Is it true? Clin Cancer Investig J 3(6) DOI: $\underline{10.4103 / 2278-0513.142652}$

22. Jogai $\mathrm{S}$ et al (2001) Acute erythroleukemia (AML-M6)-a study of clinicohematological, morphological and dysplastic features in 10 cases Ind J Can 38(2-4) 143-8

23. Keifer $\mathrm{J}$ et al (1984) Erythroleukemic infiltration of a lymph node: use of hemoglobin immunohistochemical techniques in diagnosis Hum Pathol 15(11) 1090-3 DOI: 10.1016/S0046-8177(84)80254-3 PMID: 6386660

24. Soondergaard-Petersen H (1975) The Di Guglielmo syndrome: a study of 17 cases. I. Clinical and haematological manifestations Acta Med Scand 198(3) 165-74 DOI: 10.1111/j.0954-6820.1975.tb19524.x PMID: 1058630

25. Roggli VL and Saleem A (1982) Erythroleukemia: a study of 15 cases and literature review Cancer 49(1) 101-8 PMID: $\underline{6947855}$

26. Zebisch A and Sill H (2008) Severe hemolysis as presenting sign of acute erythroleukemia $J$ Clin Oncol: official journal of the American Society of Clinical Oncology 26(2) 330-1 DOI: 10.1200/JCO.2007.14.1481

27. Mazzella FM et al (2000) The acute erythroleukemias Clin Lab Med 20(1) 119-37 PMID: 10702900

28. Wang SA et al (2008) Erythroid-predominant myelodysplastic syndromes: enumeration of blasts from nonerythroid rather than total marrow cells provides superior risk stratification Mod Pathol 21(11) 1394-402 PMID: 18839018

29. Arenillas $L$ et al (2016) Considering bone marrow blasts from nonerythroid cellularity improves the prognostic evaluation of myelodysplastic syndromes J Clin Oncol: official journal of the American Society of Clinical Oncology 34(27) 3284-92 DOI: 10.1200/ $\underline{\text { JCO.2016.66.9705 }}$

30. Domingo-Claros A et al (2002) Acute erythroid neoplastic proliferations. A biological study based on 62 patients Haematologica 87(2) 148-53 PMID: 11836165 
31. Aljabry M (2015) Complex karyotype with novel translocation in pure erythroid leukemia patient Hematol Rep 7(1) 5674 DOI: 10.4081/hr.2015.5674 PMID: 25852849 PMCID: 4378206

32. Liu W et al (2011) Pure erythroid leukemia: a reassessment of the entity using the 2008 World Health Organization classification Mod Pathol 24(3) 375-83 DOI: 10.1038/modpathol.2010.194

33. Mrózek K et al (2012) Prognostic significance of the European LeukemiaNet standardized system for reporting cytogenetic and molecular alterations in adults with acute myeloid leukemia J Clin Oncol 30(36) 4515-23 DOI: 10.1200/JCO.2012.43.4738 PMID: 22987078 PMCID: $\underline{3518729}$

34. Mrozek K (2008) Cytogenetic, molecular genetic, and clinical characteristics of acute myeloid leukemia with a complex karyotype Semin Oncol 35(4) 365-77 DOI: 10.1053/j.seminoncol.2008.04.007 PMID: 18692687 PMCID: $\underline{3640813}$ 